\title{
FMH und FMH Services an der IFAS 2010
}

Wir sind auch an der 31. Fachmesse für den Gesundheitsmarkt wieder für unsere FMH-Mitglieder da und freuen uns auf jeden persönlichen Kontakt. Kommen
Am Donnerstag, 28. Oktober 2010, haben Sie Gelegenheit, an unseren inzwischen sehr beliebten Workshops teilzunehmen. Bestimmt finden Sie in un-

\section{Besuchen Sie unsere Workshops am Donnerstag, 28.10.2010}

Sie an unseren Stand auf der Galerie vor der Halle 7, diskutieren Sie mit uns und lernen Sie die vielfältigen Dienstleistungen kennen, die Ihnen die FMH und die FMH Services bieten. serem Angebot das eine oder andere Thema, das Sie besonders interessiert. Wir freuen uns auf Ihre Anmeldung.

\section{FMH-Workshop Nr. 1: Die Health Professional Card der FMH}

Donnerstag, 28.10.2010, 10.15 Uhr-11.15 Uhr

Referent: Dr. Harald Heuser, Mitglied Projektgruppe FMH-HPC

Was ist die FMH-HPC, wie und wofür kann ich sie einsetzen? In unserem Workshop lernen Sie nicht nur die Grundfunktionalitäten der FMH-HPC (Windowsund Mac-Umgebung) kennen (Installation, elektronische Signatur, Verschlüsselung, Login-Funktionen), wir informieren Sie, entsprechend dem Entwicklungsstand, auch über die neusten Einsatzmöglichkeiten.

\section{FMH-Workshop Nr. 2: Die neue HIN Plattform - WebMail, Mobilität, HPC Einsatz und offene Standards}

Donnerstag, 28.10.2010, 11.30-12.30 Uhr

Referent: Thomas Gwerder, Leiter eHealth-Plattform, Health Info Net AC

HIN ist heute im elektronischen Datenaustausch mit über 35 Millionen Transaktionen pro Jahr der Marktführer im Schweizer Gesundheitswesen. Diese Position wird mit der Erneuerung der HIN Plattform weiter gestärkt. Die «neue HIN Plattform» erfüllt aktuelle, aber auch zukünftige Bedürfnisse der Ärzteschaft und der anderen Akteure im Gesundheitswesen. Wir stellen die neuen Möglichkeiten vor:

\section{Neuerung}

Mobiler Zugang zur HIN Plattform

Neue Secure Mail Funktionen

Einfachere Secure Mail Möglichkeiten

HIN-Abo und -Funktionen in Spitalumgebungen

Neue öffentliche HIN Zertifikate

Sicherheit und Vertraulichkeit

Krankenversicherungs-

Informationen (VVK)

\section{Nutzen und Möglichkeiten}

Mobilität wird immer mehr vorausgesetzt. Mit mobilen Zugangsmöglichkeiten erfüllt HIN dieses Bedürfnis. Der Zugang zu HIN aus jeder Ecke der Welt über Computer und Mac, aber auch über iPhone \& Co. wird dadurch Realität - ohne Software Installation.

Neue Zugangsmöglichkeiten werden sein:

- Health Professional Card (HPC) der FMH;

- SuisseID - sicherer elektronischer Identitätsnachweis als SmartCard oder USB Stick. Der Bund unterstützt die Integration bei HIN finanziell.

- SMS Code über Challenge/Respond Service (M-TAN).

HIN WebMail: Lesen und Beantworten von gesicherten E-Mails direkt über die neue Webmail Oberfläche. Nächste Ausbaustufe ab Q1/2011: Kommunikation mit Nicht-HIN-Teilnehmern (z.B. Patienten mit @bluewin.chAdresse);

Vereinfachte Anbindung von Firmen Mail-Systemen (Bsp. Kliniken und Spitäler, Versicherer, Labors usw.) über Standardkomponenten. Somit weitere Erhöhung der Reichweite für alle HIN Teilnehmerinnen und Teilnehmer. Ideal für Beleg- und Spitalärzte über mobilen Plattformzugang. Zugriff auf HIN Dienste ohne lokale Software Installation.

Ablösung der proprietären HIN Zertifikate (PKI, Public Key Infrastruktur). Weitere Erhöhung der Glaubwürdigkeit und Ermöglichung von sicherem Informationsaustausch über die Grenzen der HIN Plattform hinaus.

Einführung revisionstauglicher Audit Trails zur klaren Nachvollziehbarkeit von Transaktionen. Fokussierung auf Datenschutz und Sicherheit.

Wird in HIN Covercard Service integriert und ist somit sichergestellt. Die neue Versichertenkarte kann mit den bestehenden Covercard Lesern genutzt werden. 


\begin{abstract}
FMH-Workshop Nr. 3: Mehrwertsteuer-Checkup
Donnerstag, 28.10.2010, 13.15 Uhr-14.15 Uhr

Referenten: Daniel Müller, FMH Treuhand Services

Am 1. Januar 2010 trat das neue MWSt-Gesetz in Kraft, das auch Auswirkungen für das Unternehmen Arzt-/Gruppenpraxis hat. In diesem Workshop wollen wir Ihnen Antworten zu folgenden Fragen liefern: Bin ich (neu) mehrwertsteuerpflichtig? Mit welcher Abrechnungsmethode fahre ich besser? Welche Umsätze sind von der MWSt ausgenommen? Wo sind die MWSt-Fallstricke bei Gruppenpraxen?
\end{abstract}

\title{
FMH-Workshop Nr. 4: Pensionskasse oder Säule 3a, was ist besser?
}

Donnerstag, 28.10.2010, 14.30-15.30 Uhr

Referent: Sergio Kaufmann, FMH Insurance Services

In den meisten Beratungsgesprächen stellt sich diese Frage immer wieder. Leider werden insbesondere Ärzte in diesem Bereich von ihren Beratern ungenügend und unprofessionell beraten, obwohl deutliche Mehrwerte und Steuereinsparungen erzielt werden könnten. Eigeninteressen stehen zuweilen im Vordergrund. Im Workshop wird offen aufgezeigt, wo die Vor- und Nachteile liegen und wie die Vorsorgeformen optimal eingesetzt werden können. Selbstverständlich wird dabei auch auf die Steuerfragen detailliert eingegangen.

\section{FMH-Workshop Nr. 5: 50 - höchste Zeit für die Pensionsplanung}

Donnerstag 28.10.2010 15.45 Uhr-16.45 Uhr

Referent: Sergio Kaufmann, FMH Insurance Services

Können wir unseren Lebensstandard nach der Pensionierung halten? Wie lange muss ich noch arbeiten? Wie setze ich meine Vorsorgefranken optimal ein? Auch hier gilt: Vorbeugen ist besser als heilen. Machen Sie spätestens jetzt Ihren Vorsorge-Checkup. In der Praxis stellen wir immer wieder fest, dass diesem Thema viel zu spät die nötige Beachtung geschenkt wird. Speziell wenn man bedenkt, dass sich dieser Abschnitt über rund ein Viertel unseres gesamten Lebens erstreckt. Im Referat wird Ihnen praxisnah an Beispielen aufgezeigt, wie Sie Ihre persönliche Situation optimieren können.

\section{FMH-Workshop Nr. 6: Der Lebenszyklus einer Arztrechnung}

Donnerstag 28.10.2010 17.00 Uhr-18.00 Uhr

Referenten: Adolf Saurer, FMH Inkasso Services / INKAS und Dirk Clüsserath, FMH Factoring Services / mediserv

Das «Leben» einer Arztrechnung durchläuft mehrere Phasen und stellt dadurch das Forderungsmanagement der Praxisadministration vor vielfältige Herausforderungen. Anbieter mit Kernkenntnissen genau in diesen Bereichen verschaffen den Praxen oft Abhilfe. Dieser Workshop klärt, welche Schwierigkeiten das Einbringen der Honorare ergibt und wie externe Partner diese Probleme professionell managen können.

\section{Weitere Informationen}

Veranstaltungsort: Die FMH-Workshops werden durchgeführt im Seminarraum K6 (Zugang vom Galeriegeschoss vor Halle 7).

Teilnehmerkreis: Die FMH-Workshops stehen ausschliesslich Ärztinnen und Ärzten sowie deren MPAs offen (sofern die Anmeldung durch den Praxisinhaber erfolgt).

Kosten: Für FMH-Mitglieder und deren MPAs (sofern durch das FMH-Mitglied angemeldet) ist die Teilnahme an den Workshops gratis. (Nicht-FMH-Mitglie- der und deren MPAs bezahlen einen Unkostenbeitrag von 30 Franken pro Workshop.)

Die Workshops werden ausschliesslich in deutscher Sprache durchgeführt.

Die Teilnehmerzahl ist begrenzt. Die Registrierung erfolgt nach Eingang der Anmeldungen.

Die Teilnahmebestätigung berechtigt zum Gratiseintritt an die IFAS 2010.

Anmeldung: Online-Anmeldeformular unter www.fmh.ch 